1 Universidade do Estado de Minas Gerais (Uemg) Divinópolis (MG), Brasil. heulerandrade@gmail.com

2 Universidade Federal de São João Del Rei (UFSJ) Divinópolis (MG), Brasil. valeriaoliveira@ufsj.edu.br

3 Universidade Federal de São João Del Rei (UFSJ) Divinópolis (MG), Brasil. enftarcisio@ufsj.edu.br

${ }^{4}$ Universidade Federal de São João Del Rei (UFSJ) Divinópolis (MG), Brasil. marcotuliocorreapessoa@ gmail.com

5 Universidade Federal de São João Del Rei (UFSJ) Divinópolis (MG), Brasil. elietealbano@ufsj.edu.br

\section{Avaliação do Programa de Controle da Tuberculose: um estudo de caso}

\author{
Evaluation of Tuberculosis Control Program: a case report
}

Heuler Souza Andrade1, Valéria Conceição Oliveira2 ${ }^{2}$, Tarcísio Laerte Gontijo ${ }^{\mathbf{3}}$, Marco Túlio Corrêa Pessôa4, Eliete Albano de Azevedo Guimarães ${ }^{\mathbf{5}}$

RESUMO A falta de integração entre os serviços que compõem a rede de atenção à saúde, além da incapacidade de muitos profissionais, são apontadas como causas da problemática para o controle da tuberculose no Brasil. Este estudo avaliou o grau de implantação do Programa de Controle de Tuberculose (PCT) em Divinópolis (MG). Trata-se de uma pesquisa avaliativa na perspectiva de análise de implantação. O PCT encontra-se parcialmente implantado. Como limitações, destacam-se a deficiência de gestão e planejamento, e a insuficiência de profissionais capacitados e insumos. Sugere-se a descentralização das ações do PCT e o aumento das ações de vigilância como possíveis soluções para a melhoria da assistência e da prevenção.

PALAVRAS-CHAVE Tuberculose. Atenção Primária à Saúde. Avaliação de programas e projetos de saúde.

\begin{abstract}
The lack of integration between health care services network, in addition to the inefficiency of health professionals, are some of the identified problems for the control of Tuberculosis in Brazil. This study evaluated the level of implementation of the Tuberculosis Control Program (PCT) in Divinopolis (MG). The results signalize that PCT is partially implemented, due mostly to deficiencies related to management and planning, the insufficiency of trained professionals and insufficient resources. The decentralization of PCT actions and the enhancement of surveillance activities are signalized as possible solutions for the improvement of TB health care and prevention.
\end{abstract}

KEYWORDS Tuberculosis. Primary Health Care. Program evaluation and health projects. 


\section{Introdução}

A tuberculose (TB) atinge a humanidade há milhares de anos, persistindo como um dos principais problemas de saúde a serem enfrentados em todo o mundo. Os determinantes socioeconômicos, juntamente com a instabilidade política, os conflitos internos e as guerras, têm promovido uma migração humana em nível internacional, contribuindo substancialmente para a disseminação da infecção em diversos países (DARA ET AL., 2012).

No cenário mundial, a incidência notificada de TB, em 2013, foi de 126 casos/100 mil habitantes, com uma estimativa de 9 milhões de casos novos e 1,5 milhões de mortes (WHO, 2014). No Brasil, em 2014, foram diagnosticados 67.966 casos novos de TB, sendo a taxa de incidência de 33,5 casos/100 mil habitantes. O País, juntamente com outros 21 países em desenvolvimento, agrega $80 \%$ dos casos mundiais da doença. No Brasil, ela é a quarta causa de mortes por doenças infecciosas e a primeira entre as doenças infecciosas definidas dos pacientes com Síndrome da Imunodeficiência Adquirida (Aids) (WHO, 2014; BRASIL, 2015).

$\mathrm{Na}$ intenção de reduzir a incidência da doença, foi implantado pelo Ministério da Saúde, no Brasil, o Programa Nacional de Controle da Tuberculose (PNCT), que preconiza a horizontalização das medidas de controle, vigilância, prevenção e tratamento da doença para a Atenção Primária à Saúde (APS), visando, principalmente, aumentar a adesão dos pacientes, a descoberta das fontes de infecção (pacientes pulmonares bacilíferos) e a cura, para reduzir o risco de transmissão da doença na comunidade. Essa estratégia de organização ampliou o acesso das populações mais vulneráveis ou sob risco acrescido de contrair a doença, pois lida diretamente com o usuário e seu contexto de vida. Atualmente, o PNCT busca aprimorar o planejamento e a avaliação das ações de controle da TB e, para isso, estruturou sua organização com os seguintes componentes e subcomponentes: atenção à saúde (prevenção, diagnóstico, assistência) e informação estratégica (vigilância epidemiológica, monitoramento e avaliação, pesquisa, desenvolvimento humano e institucional, comunicação e mobilização social, planejamento e orçamento) (BRASIL, 2011; HEUFEMANN; GONÇALVES; GARNELO, 2013).

Em Divinópolis (MG), o Programa de Controle da Tuberculose (PCT) foi descentralizado para a APS em 2012. Essa intervenção reforça a importância do cuidado com os portadores de TB na comunidade, incluindo, no cotidiano dos serviços, ações preventivas e de controle da doença. Nesta perspectiva, faz-se necessária uma sistematização das atividades, com um formato de gestão integrado entre os níveis de atenção e com profissionais comprometidos com as demandas do portador de TB (SILVA et AL., 2014). Agregamse a isto, adequação quanto às instalações e equipamentos, apoio laboratorial, recursos humanos (como o número, a variedade e a qualificação dos profissionais), medicamentos tuberculostáticos, protocolo, sistema de informação, recursos materiais e recursos financeiros (BRASIL, 2011).

Estudos que avaliaram o PNCT têm evidenciado que os serviços de saúde, no Brasil, não estão adequadamente preparados para assistir ao portador de TB. Problemas como a falta de integração entre os serviços que compõem a rede de atenção à saúde, baixa resolutividade da APS no diagnóstico, falhas na retaguarda laboratorial e a incapacidade profissional ao lidar com novas tecnologias são apontados como causas da atual situação da doença no País (PONCE ET AL., 2013; SCATENA ET AL., 2009; VILLA ET AL., 2013; SILVA ET AL., 2014).

Embora se considere relevante a problemática em torno da atuação dos serviços de saúde para o controle da TB, ainda são escassos os estudos avaliativos sobre a implantação de PCTs. As pesquisas existentes têm colaborado para fortalecer a adesão dos municípios ao programa, mas os avanços verificados ainda são insuficientes para o 
controle da TB nas localidades (HEUFEMANN; GONÇALVES; GARNELO, 2013; SILVA ET AL., 2014; OLIVEIRA; NATAL; CAMACHO, 2015).

Os estudos avaliativos constituem-se como ferramentas valiosas para subsidiar processos de mudança na organização dos serviços, na medida em que proporcionam, aos envolvidos ou interessados, direta ou indiretamente, condições para decidir como enfrentar e resolver problemas no cotidiano dos serviços de saúde (CHAMPAGNE ET AL., 2011). Avaliar a implantação do PCT no componente da atenção à saúde em Divinópolis (MG) proporcionará apoio aos gestores, no sentido de organizar a assistência na APS. Em 2012, a taxa de incidência da TB nesse município foi de 12,9 casos/100 mil habitantes, enquanto a taxa de mortalidade foi de 1,4 óbitos/100 mil habitantes, e os índices de cura e abandono do tratamento foram de $57,1 \%$ e 14,3\%, respectivamente. Embora esteja abaixo dos valores nacionais, a taxa de incidência do município ainda é considerada alta em relação aos parâmetros preconizados pela Organização Mundial da Saúde (OMS) (SILVA, 2014).

Este estudo teve como objetivo avaliar o grau de implantação do PCT, no componente da atenção à saúde, em Divinópolis (MG).

\section{Material e métodos}

Trata-se de uma pesquisa avaliativa, com abordagem quantitativa, que analisou a implantação do PCT em Divinópolis (MG), no período entre 2014 e 2015. Optou-se pelo estudo de caso (YIN, 2010), propício para a análise de implantação de programas, no qual as explicações dos fatos decorrem da profundidade da análise do caso, e que consiste em especificar o conjunto dos fatores estruturais e de processo que facilitam ou comprometem os resultados obtidos na intervenção. O questionamento sobre a validade externa, deste tipo de estudo, pode ser colocado como uma importante limitação quando da observação de uma única situação (CHAMPAGNE ET AL., 2011).
Divinópolis situa-se na região CentroOeste de Minas Gerais e encontra-se entre os dez principais municípios do estado. Possui uma população estimada em 230.848 habitantes e o seu sistema de saúde é composto por 32 equipes de Estratégia de Saúde da Família (ESF). Além disso, conta com dez unidades básicas de saúde tradicional (USTs), uma policlínica, um centro de apoio diagnóstico, um centro regional de reabilitação, um ambulatório de apoio a doenças endêmicas, um serviço de referência em saúde mental, um serviço especializado em odontologia, um laboratório municipal, uma unidade de pronto atendimento municipal e quatro hospitais, sendo um filantrópico e três privados. A cobertura de ESF do município, em abril de 2015 , foi de $50,78 \%$.

O caso selecionado foi o do município cuja unidade de análise foi o PCT em seu componente atenção à saúde, que reforça a importância do cuidado aos portadores de TB na APS. O modelo lógico (figura 1) do PCT, adaptado de Oliveira e Natal (2007), contempla: insumos, atividades, produtos, resultados e impacto do programa avaliado.

Quanto aos insumos, priorizou-se o apoio laboratorial, baseado na realização de baciloscopia de escarro, cultura de escarro e teste do vírus da imunodeficiência humana (HIV); a medicação para TB; o profissional qualificado e capacitado regularmente, atualizado sobre as recomendações de TB; a normatização de condutas; e o Sistema de Informação de Agravos de Notificação (Sinan).

Em relação às atividades, foi dada prioridade à realização dos exames em tempo hábil, à realização de exame nos contatos e ao fluxo de entrega desses exames. Quanto aos profissionais, buscou-se conhecer as ações de atenção aos portadores de TB, o acompanhamento das recomendações para diagnóstico, o tratamento e a prevenção, além da realização de capacitação e treinamento para os técnicos. Em relação aos documentos, considerou-se o uso de manuais e normas específicas do PNCT para as condutas junto 
ao portador de TB, a existência de impressos específicos para registros e notificações, bem como o fluxo dessas informações.

No que diz respeito aos produtos, a partir das atividades realizadas, as pessoas com sintomas respiratórios devem ser registradas e examinadas, submetidas à baciloscopias e à cultura de escarro. Os profissionais de saúde devem registrar e notificar os casos novos de $\mathrm{TB}$ e as informações devem ser confiáveis. Os portadores de TB devem ser acompanhados através do Tratamento Diretamente Observado (TDO), e os contatos, monitorados.
Quanto aos resultados, espera-se que um maior número de portadores de TB seja diagnosticado precocemente, havendo, assim, uma interrupção da cadeia de transmissão da doença; que haja maior eficácia no acompanhamento da infecção HIV/TB; que haja aumento das taxas de cura e diminuição das taxas de abandono; e que a qualidade das informações melhore. Em relação ao impacto, espera-se uma redução da incidência e da mortalidade por TB. Esses dois últimos efeitos desejados não foram avaliados nesta pesquisa.

Figura 1. Programa de controle e tratamento da tuberculose no município de Divinópolis (MG)

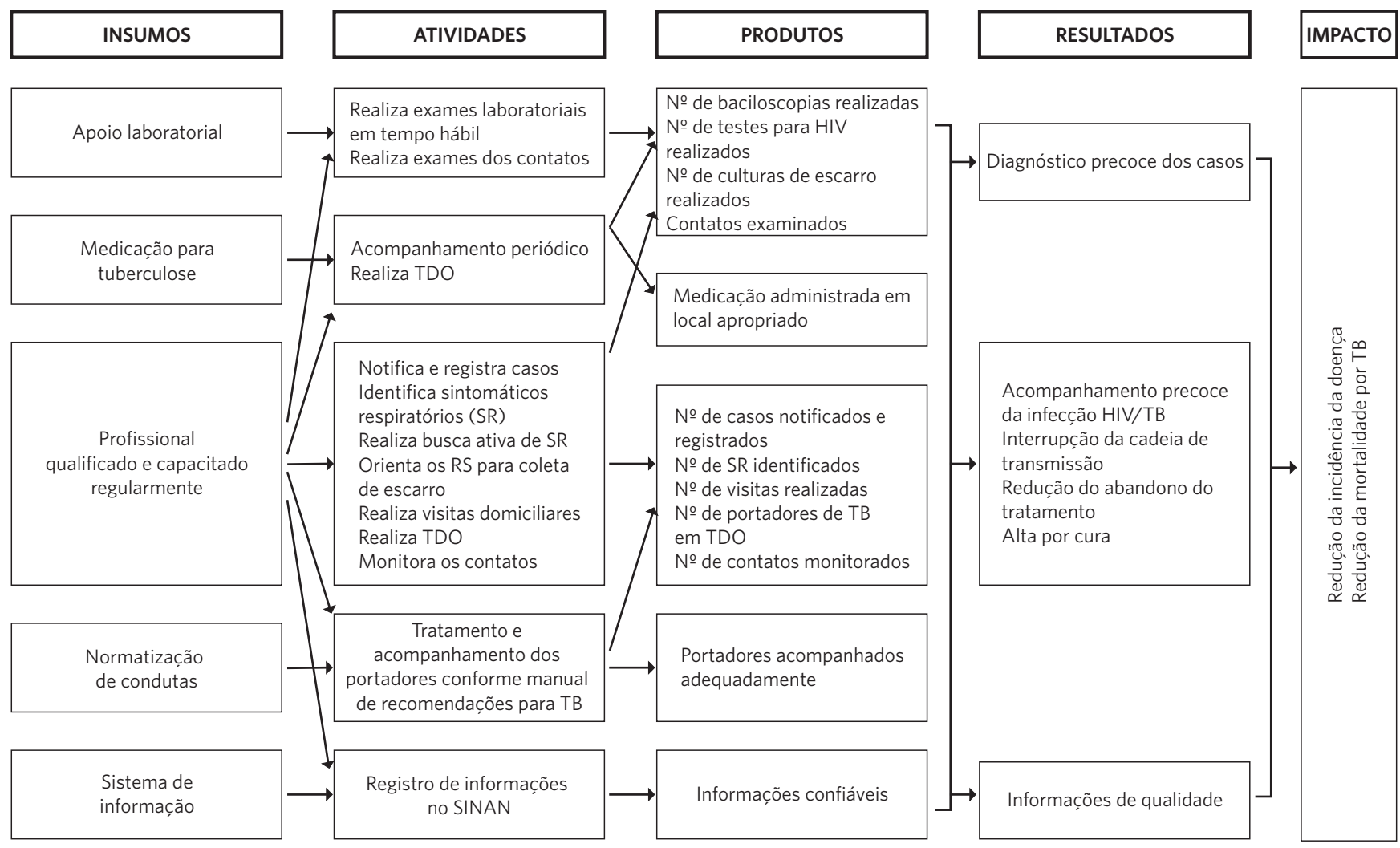


Foram convidados a participar do estudo os profissionais - agentes comunitários de saúde (ACSs), enfermeiros, médicos e técnicos de enfermagem - da APS (ESF e UST), profissionais de referência do programa e portadores de TB. O contato com os interessados foi realizado, primeiramente, por telefone, para facilitar o encontro pessoal. Posteriormente, foi realizada a entrega dos questionários, que os profissionais de referência do PCT e da APS receberam, pessoalmente, nos locais de trabalho. Os portadores de TB os receberam no próprio domicílio. Foram realizadas três visitas à coordenação do PCT, três visitas a cada unidade de APS e três visitas ao domicílio de cada portador. Foram desenvolvidas, ainda, análises de bases de dados nacionais e de documentos, como manuais técnicos, portarias e livros de registros de pacientes. A coleta de dados ocorreu entre novembro e dezembro de 2014.

Foram utilizados dois questionários estruturados, autopreenchidos, direcionados aos responsáveis pelo $\mathrm{PCT}$, aos profissionais da assistência; e um questionário aplicado aos portadores de TB. Os responsáveis pelo PCT responderam a um questionário referente ao contexto organizacional, enquanto os profissionais da assistência responderam a perguntas sobre o contexto técnico. O questionário dos portadores de TB foi relacionado ao contexto de efeito. As informações do contexto externo foram obtidas na base de dados do Instituto Brasileiro de Geografia e Estatística (IBGE), referentes ao censo do ano de 2010. Os questionários foram submetidos a um pré-teste para verificação e adequação de possíveis erros. Os aplicadores foram os próprios pesquisadores.

Considerou-se, como contexto interno, o programa propriamente dito, que integra os contextos organizacional, técnico e efeito. E, como vulnerabilidade, o contexto externo ao programa. Pretendeu-se avaliar as influências dos contextos interno e externo no grau de implantação do PCT.

No contexto organizacional, as categorias analisadas foram Gestão e Planejamento, Serviços de Referência e Insumos, e Vigilância Epidemiológica, que se referem às características de gestão do programa na secretaria de saúde e à relação da gestão com as unidades de saúde. O contexto técnico refere-se à maneira como as ações de saúde são realizadas pelas equipes das ESFs e das USTs. As categorias avaliadas foram Diagnóstico, Assistência e Prevenção, e os seus respectivos critérios. O contexto de efeito analisa a qualidade da atenção sob a opinião do portador em relação à acessibilidade, ao diagnóstico, à assistência e à prevenção. $\mathrm{O}$ contexto externo refere-se à vulnerabilidade social do indivíduo morador do município de Divinópolis (MG). Os critérios utilizados foram renda por pessoa e grau de escolaridade.

Para classificar o grau de implantação do PCT, foram construídas, a partir do modelo lógico, matrizes de análise e julgamento. As matrizes de análise, adaptadas do estudo de Gutierrez (2012), foram consensuadas entre os envolvidos na avaliação e os pesquisadores externos, pois as condições culturais e o modelo de atenção à saúde se diferenciam, podendo interferir na qualidade do dado. Cada dimensão dos contextos organizacional, técnico e de efeito foi subdividida em categorias, e estas foram constituídas por critérios. Foi atribuída uma pontuação máxima, entre 5 e 10 pontos, para todos os itens avaliados, mediante a relevância de cada critério.

O grau de implantação do programa foi definido por meio de um sistema de escores, com pesos diferenciados para cada indicador e/ou critério dos contextos organizacional, técnico e efeito, segundo o nível de importância atribuído. Foi estabelecida a pontuação máxima - 525 pontos -, distribuída entre as dimensões do contexto interno. $\mathrm{O}$ contexto organizacional recebeu o máximo de 250 pontos, distribuídos entre as categorias Gestão e Planejamento (110), Serviços de Referências e Insumos (90) e 
Vigilância Epidemiológica (50). O contexto técnico recebeu o máximo de 150 pontos, distribuídos entre as categorias Diagnóstico (45), Assistência (60) e Prevenção (45). O contexto de efeito recebeu o máximo de 125 pontos, distribuídos entre as categorias Acessibilidade (30), Diagnóstico (35), Assistência (45) e Prevenção (15). Os critérios do contexto externo (vulnerabilidade) somaram 15 pontos, distribuídos nas categorias Renda por Pessoa (10) e Grau de Escolaridade (5).

Para a construção dos escores do grau de implantação, inicialmente, foram determinados os valores observados ( $\Sigma$ dos pontos dos indicadores) e calculado o grau de implantação, em termos percentuais ( $\Sigma$ observados $\div$ $\Sigma$ das pontuações máximas possíveis $\times 100$ ) para cada componente, e, posteriormente, realizou-se a somatória dos componentes para o cálculo do grau de implantação total. A partir desses percentuais, definiu-se a classificação para o grau de implantação: Adequado (76\% a $100 \%$ ), Parcialmente Adequado (51\% a 75\%), Incipiente (26\% a $50 \%$ ) e Não Adequado ( $0 \%$ a $25 \%$ ). As medidas-síntese e de posição foram utilizadas na avaliação das categorias de cada dimensão. Os dados foram tratados no Microsoft Excel ${ }^{\circledR} 2013$, processados e tabulados no Statistical Pacckage for the Social Sciences $\left(\right.$ SPSS $\left.^{\circledR}\right)$ 20.0.

Este estudo obedeceu aos princípios éticos da Resolução ${ }^{0}$ 466/12, do Conselho Nacional de Saúde, e foi aprovado pela Secretaria Municipal de Saúde e junto ao Comitê de Ética em Pesquisa, Certificado de Apresentação para Apresentação Ética (CAAE) 34225214.7.0000.5545, parecer 751.699 .

\section{Resultados}

A população elegível para o estudo foi de 340 pessoas, sendo 322 profissionais da APS (ESF e UST), 4 profissionais de referência do programa e 14 portadores de TB em tratamento. Dos 326 profissionais convidados a responder os questionários, 37 (11,35\%) se recusaram a participar e 48 (14,7\%) não se manifestaram, sendo excluídos da pesquisa. Demonstraram interesse em participar das etapas da pesquisa 241 (73,9\%) profissionais. Destes, 119 (36,5\%) responderam os questionários. As perdas foram consideradas após três tentativas para a aplicação dos questionários. Dos 119 profissionais que responderam ao questionário, 75 (63\%) trabalhavam em ESFs. Destes, 22 (29,3\%) eram enfermeiros, 15 (20\%) eram médicos, 14 (18,7\%) eram técnicos ou auxiliares de enfermagem e 24 (32\%) eram ACSs. Dos 40 profissionais que trabalhavam em USTs, 15 (37,5\%) eram enfermeiros, 4 (10\%) eram médicos e 21 (52,5\%) eram técnicos ou auxiliares de enfermagem. Dos 14 portadores em tratamento, 3 (21,43\%) estavam sendo acompanhados por profissionais da rede privada e não utilizavam os serviços da APS, tendo sido excluídos. Os demais portadores (11) consentiram em participar do estudo. Sendo assim, 130 pessoas fizeram parte desta pesquisa.

No contexto organizacional (tabela 1), verificou-se a existência de profissionais que atuavam na organização do PCT (100\%), contudo estes possuíam tempo de gerência inferior a dois anos (20\%) e capacitação insuficiente para o desenvolvimento das ações de gerenciamento (66,7\%). Na gestão, observou-se deficiência quanto à realização de reuniões para discutir e programar ações de controle da TB $(33,3 \%)$ e quanto à supervisão da equipe de coordenação do PCT, que não atendia às necessidades das unidades de saúde (33,3\%). Além destes, destacaram-se, como pontos dificultadores, a escassez de planejamento entre a equipe técnica do PCT com participação dos gerentes das unidades; a baixa incidência de ações de supervisão realizadas pelos coordenadores do PCT; a inexistência de um fluxo padronizado das informações do Sinan; e a falta de relatórios sobre a situação da TB.

Quanto à categoria Serviços de Referência 
e Insumos, observou-se que, embora se tenha afirmado que a equipe técnica utilizava normas do PNCT (100\%), existiam deficiências de várias ações, como a definição para referência e contrarreferência para portadores de tuberculose $(66,7 \%)$ e a existência de estratégias definidas para portadores de TB em abandono de tratamento (66,7\%). Outros dois critérios apresentaram-se como inexistentes: a análise dos mapas de medicamentos das unidades e a predefinição do fluxo para os portadores realizarem prova tuberculínica. Sobre os insumos, os resultados apontaram que os serviços tinham fichas de notificação e investigação da TB (100\%), mas que faltava organização no envio de coletor de escarro para as unidades de saúde (66,7\%) e na distribuição de medicamentos, que não era realizada conforme o preconizado pelo $\operatorname{PNCT}(33,3 \%)$.
A Vigilância Epidemiológica foi a categoria de menor classificação. A frequência do fluxo de informações entre as unidades de saúde e a Secretaria Municipal de Saúde (SMS) não foram bem definidas (66,7\%), assim como a realização de ações de Vigilância Epidemiológica, que se mostraram deficientes (66,7\%). Observou-se que as informações eram coletadas nos serviços da APS ou nos serviços de média complexidade, mediante a notificação do caso. Os formulários de notificação e investigação seguiam para a SMS, onde eram processadas as informações no Sinan e repassadas, semanalmente, à Superintendência Regional de Saúde (SRS). Os maiores entraves desta categoria foram a inexistência de ações importantes, como o desconhecimento da chegada da notificação oriunda das unidades de APS e a periodicidade de atualização do livro de registros de casos de TB.

Tabela 1. Grau de implantação dos critérios avaliados no contexto organizacional do PCT, Divinópolis (MG). Brasil, 2014-2015

\begin{tabular}{lcc}
\hline Categorias/Critérios & $\begin{array}{c}\text { Pontuação } \\
\text { máxima esperada }\end{array}$ & $\begin{array}{c}\text { Grau de } \\
\text { implantação }\end{array}$ \\
\hline Gestão e Planejamento & 10 & 20 \\
\hline Tempo de gerência & 10 & 66,7 \\
Existência de planejamento entre a equipe técnica do PCT, com participa- & 10 & 33,3 \\
ção dos gerentes das unidades & 10 & 66,7 \\
Realização de reuniões para discutir e programar ações de controle da TB & 10 & 66,7 \\
Realização de ações de supervisão das ações de controle da TB & 10 & 66,7 \\
Realização de capacitações para profissionais de saúde & 10 & 10,7 \\
Existência de fluxo padronizado das informações do Sinan & 10 & 100 \\
Equipe técnica do PCT faz relatório sobre a TB & 10 & 100 \\
Equipe técnica conhece a proporção de unidades de saúde que atendem & 10 & 100 \\
TB & 10 & 33,3 \\
Área territorial das unidades bem definida & 110 & $\mathbf{6 5 , 5}$ \\
Número de profissionais que atuam com TB & & 10 \\
Supervisão do PCT atende às necessidades das unidades & & 10 \\
\hline Total & & 10 \\
\hline
\end{tabular}




\begin{tabular}{|c|c|c|}
\hline \multicolumn{3}{|l|}{ Serviços de Referência e Insumos } \\
\hline Equipe faz análise dos mapas de medicamentos das unidades & 10 & 0 \\
\hline Equipe utiliza normas do PNCT & 10 & 100 \\
\hline Distribuição de medicamentos de TB para as unidades & 10 & 33,3 \\
\hline Predefinição do fluxo para os portadores realizarem prova tuberculínica & 10 & 0 \\
\hline Existência de referência e contra referência para portadores de TB & 10 & 66,7 \\
\hline $\begin{array}{l}\text { Existência de estratégias definidas, para as unidades, para registro e atua- } \\
\text { ção em relação a contatos com a TB }\end{array}$ & 10 & 66,7 \\
\hline $\begin{array}{l}\text { Existência de estratégias definidas para portadores de TB em abandono de } \\
\text { tratamento }\end{array}$ & 10 & 66,7 \\
\hline Existência de fichas de notificação e investigação epidemiológicas & 10 & 100 \\
\hline Envio de coletor de escarro para as unidades & 10 & 66,7 \\
\hline Total & 90 & 55,6 \\
\hline \multicolumn{3}{|l|}{ Vigilância Epidemiológica } \\
\hline $\begin{array}{l}\text { Existência de profissionais capacitados para realizar o acompanhamento } \\
\text { das informações no Sinan }\end{array}$ & 10 & 100 \\
\hline $\begin{array}{l}\text { Boa definição da frequência do fluxo de informações entre as unidades de } \\
\text { saúde e a SMS }\end{array}$ & 10 & 66,7 \\
\hline Monitoramento de ações de vigilância epidemiológica & 10 & 66,7 \\
\hline Periodicidade da notificação oriunda das unidades de saúde & 10 & 0 \\
\hline Periodicidade da atualização do livro de registro de casos de TB & 10 & 0 \\
\hline Total & 50 & 46,7 \\
\hline
\end{tabular}

Fonte: Elaboração própria.

Nocontextotécnico (tabela2), observou-se insuficiência quanto à realização de radiografia de tórax (64,66\%), e quanto ao tempo entre a coleta e a chegada do resultado de baciloscopia de escarro $(39,14 \%)$. Os serviços de apoio diagnóstico dispunham de um Centro de Apoio à Saúde, onde estava inserido o Laboratório Municipal, responsável pela realização dos exames de baciloscopia, cultura de escarro e radiológicos, utilizados na definição da TB.

Todas as ações assistenciais denotaram problemas. Destaca-se a falta de medicação no último ano (61,2\%), a realização deficiente do TDO (51,72\%), o baixo número de consultas durante o tratamento $(57,24 \%)$ e o tempo de espera do portador de TB para ser atendido no dia da consulta (51,6\%), que foi, em média, superior a uma hora. E ainda, somente $12,1 \%$ dos medicamentos foram entregues na APS. A assistência farmacêutica era garantida pela Farmácia Municipal Central, responsável pela dispensação de medicamentos que eram liberados a partir 
da notificação do caso confirmado, mediante formulário de investigação devidamente preenchido.

$\mathrm{Na}$ prevenção, observou-se falta de agendamento para realização de visitas domiciliares (52,58\%), escassez de ações comunicativas de prevenção $(33,62 \%)$ e realização periódica da busca de casos novos (55,17\%).

Tabela 2. Grau de implantação dos critérios avaliados no contexto técnico do PCT, Divinópolis (MG). Brasil, 2014-2015

\begin{tabular}{|c|c|c|}
\hline Categorias/Critérios & $\begin{array}{l}\text { Pontuação } \\
\text { máxima esperada }\end{array}$ & $\begin{array}{l}\text { Grau de } \\
\text { implantação }\end{array}$ \\
\hline \multicolumn{3}{|l|}{ Diagnóstico } \\
\hline Sintomáticos respiratórios são submetidos a baciloscopia de escarro & 10 & 80,17 \\
\hline Existência de oferta de teste anti-HIV & 10 & 79,31 \\
\hline $\begin{array}{l}\text { Tempo entre coleta e chegada de resultado de baciloscopia de escarro, nas } \\
\text { unidades de saúde }\end{array}$ & 5 & 39,14 \\
\hline Realização de radiografia de tórax & 10 & 64,66 \\
\hline Notificação de casos de TB pela unidade & 10 & 83,62 \\
\hline Total & 45 & 72,7 \\
\hline \multicolumn{3}{|l|}{ Assistência } \\
\hline Local de dispensação de medicamentos & 5 & 12,1 \\
\hline Falta de medicação no último ano & 5 & 61,2 \\
\hline Tempo de espera do doente de TB, para ser atendido no dia da consulta & 5 & 51,6 \\
\hline Atendimento do doente de TB pelo mesmo profissional & 5 & 62,93 \\
\hline Número de consultas durante o tratamento & 10 & 57,24 \\
\hline Utilização de normas do PNCT & 10 & 91,4 \\
\hline Preenchimento dos livros de registros de casos de TB & 10 & 44,83 \\
\hline Oferecimento do TDO aos portadores novos & 10 & 51,72 \\
\hline Total & 60 & 56,5 \\
\hline \multicolumn{3}{|l|}{ Prevenção } \\
\hline Realização periódica da busca de casos novos & 10 & 55,17 \\
\hline Realização de ações de prevenção - comunicação em saúde & 10 & 33,62 \\
\hline Existência de agendamento para realização de visitas domiciliares & 5 & 52,58 \\
\hline Realização de prova tuberculínica (PT) & 10 & 77,59 \\
\hline Contato submetido a consulta médica/enfermagem & 10 & 77,59 \\
\hline Total & 45 & 60,1 \\
\hline
\end{tabular}


A Acessibilidade foi a categoria mais bem avaliada no contexto de efeito, considerada adequada. No Diagnóstico, os principais entraves foram os critérios que diziam respeito ao fornecimento de coletor de escarro para diagnóstico $(11,1 \%)$ e ao número de amostras de escarro coletadas para diagnóstico (50\%), que foi, em média, de uma amostra. Sobre a Assistência, notou-se que os pacientes esperaram menos de 30 minutos para a consulta médica (100\%), enquanto que, para a consulta de enfermagem, esse tempo foi, em média, superior a uma hora (33,3\%). Em relação ao esclarecimento das dúvidas dos pacientes, nem todos os profissionais exerceram esta atividade $(37,8 \%)$, assim como, a poucos pacientes foi oferecido o TDO (11,1\%). Na categoria Prevenção, foi observado que, embora os contatos fossem convocados para exames (77,8\%), um número bem menor era examinado $(55,6 \%)$ e poucos receberam visita domiciliar (66,7\%). O contexto externo foi avaliado como incipiente, com implantação de 46,5\%. Os resultados mostraram que a renda da população do município estava abaixo de três salários mínimos, enquanto a escolaridade se apresentou melhor, tendo, a maior parte das pessoas, mais de oito anos de estudo (tabela 3).

Tabela 3. Grau de implantação dos critérios avaliados nos contextos de efeito e externo do PCT, Divinópolis (MG). Brasil, 2014-2015

\begin{tabular}{|c|c|c|}
\hline Categorias/Critérios & $\begin{array}{c}\text { Pontuação } \\
\text { máxima esperada }\end{array}$ & $\begin{array}{c}\text { Grau de } \\
\text { implantação }\end{array}$ \\
\hline \multicolumn{3}{|l|}{ Contexto de Efeito } \\
\hline \multicolumn{3}{|l|}{ Acessibilidade } \\
\hline Tempo de utilização da unidade de saúde & 10 & 78,9 \\
\hline Passível de mudança para outra unidade de saúde & 5 & 100 \\
\hline Tempo percorrido para chegar à unidade de saúde & 10 & 94,4 \\
\hline Usuário perde o dia de trabalho para ir à unidade de saúde & 5 & 88,9 \\
\hline Total & 30 & 89,3 \\
\hline \multicolumn{3}{|l|}{ Diagnóstico } \\
\hline Tempo levado para conhecimento do diagnóstico após o início dos sintomas & 10 & 71,1 \\
\hline Oferecimento do teste HIV & 5 & 66,7 \\
\hline Realização do exame de escarro para diagnóstico & 5 & 55,6 \\
\hline Número de amostras de escarro coletadas para diagnóstico & 10 & 50 \\
\hline Fornecimento, pela unidade, de coletor de escarro para diagnóstico & 5 & 11,1 \\
\hline Total & 35 & 53,7 \\
\hline \multicolumn{3}{|l|}{ Assistência } \\
\hline Dificuldades para marcação da consulta & 10 & 88,9 \\
\hline Tempo de espera para consulta com o médico & 10 & 100 \\
\hline Tempo de espera para consulta com enfermeiro & 10 & 33,3 \\
\hline Profissional que esclarece dúvidas & 10 & 37,8 \\
\hline Oferecimento de TDO & 5 & 11,1 \\
\hline Total & 45 & 59 \\
\hline
\end{tabular}


Tabela 3. (cont.)

\begin{tabular}{lcr}
\hline Prevenção & & \\
\hline Convocação de contatos para exames & 5 & 77,8 \\
Realização de exame para os contatos & 5 & 55,6 \\
Recebimento de visita domiciliar & 5 & $\mathbf{6 6 , 7}$ \\
\hline Total & & $\mathbf{6 6 , 7}$ \\
\hline Contexto Externo & 15 & 25,47 \\
\hline Vulnerabilidade & 5 & 85,05 \\
\hline Renda por pessoa & $\mathbf{1 5}$ & $\mathbf{4 6 , 5}$ \\
\hline Escolaridade & & \\
\hline Total & & \\
\hline
\end{tabular}

Fonte: Elaboração própria.

O PCT, em Divinópolis (MG), encontra-se parcialmente implantado, com classificação de $61,8 \%$. Entre os contextos avaliados, verificou-se que o organizacional obteve a menor classificação, com $58,1 \%$, sendo este valor influenciado, principalmente, pelas categorias Vigilância Epidemiológica (46,7\%) e Serviços de Referência e Insumos (55\%). Os contextos técnico e de efeito foram pontuados, respectivamente, em
$61,7 \%$ e $65,7 \%$. A categoria mais bem avaliada no contexto técnico foi o Diagnóstico (72,7\%), enquanto a Assistência obteve pontuação mais baixa (56,5\%). Quanto à opinião que os portadores tinham, em relação à qualidade da atenção do PCT, verificou-se que a acessibilidade foi bem avaliada (89,3\%). Contudo, problemas foram identificados nas categorias de Diagnóstico, Assistência e Prevenção (tabela 4).

Tabela 4. Grau de implantação do PCT de Divinópolis (MG), segundo os contextos organizacional, técnico e efeito. Brasil, 2014-2015

\begin{tabular}{lrr}
\hline Contextos & $\begin{array}{c}\text { Pontuação } \\
\text { máxima esperada }\end{array}$ & $\begin{array}{c}\text { Grau de } \\
\text { implantação }\end{array}$ \\
\hline Organizacional & $\mathbf{2 5 0}$ & $\mathbf{5 8 , 1}$ \\
\hline Gestão e Planejamento & 110 & $\mathbf{6 5 , 5}$ \\
Serviços de Referência e Insumos & 90 & 55 \\
Vigilância Epidemiológica & 50 & $\mathbf{6 2 , 4}$ \\
\hline Técnico & $\mathbf{1 5 0}$ & $\mathbf{7 2 , 7}$ \\
\hline Diagnóstico & 45 & 56,5 \\
Assistência & 60 & 60,1 \\
Prevenção & 45 & 45 \\
\hline
\end{tabular}


Tabela 3. (cont.)

\begin{tabular}{lcr}
\hline Efeito & $\mathbf{1 2 5}$ & $\mathbf{6 5 , 7}$ \\
\hline Acessibilidade & 30 & 89,3 \\
Diagnóstico & 35 & 53,7 \\
Assistência & 45 & 59 \\
Prevenção & 15 & 66,7 \\
\hline Total & $\mathbf{5 2 5}$ & $\mathbf{6 1 , 8}$ \\
\hline
\end{tabular}

Fonte: Elaboração própria.

\section{Discussão}

Os resultados desfavoráveis para a implantação do PCT em Divinópolis (MG) evidenciaram que muitos critérios avaliados nos contextos organizacional e técnico influenciaram a implantação do programa. A maneira como o PCT está disposto no cenário municipal, especialmente no que diz respeito à descentralização parcial de suas ações para a APS e a deficiência de gestão, repercutiram nas ações de assistência ao portador de TB, na vigilância epidemiológica e na prevenção e promoção à saúde.

A forma de gerenciamento do PCT municipal demonstra que há um descompasso entre o processo de gerenciamento do programa e suas ações. O pouco tempo dos coordenadores no cargo de gerência pode estar causando falta de planejamento de ações de controle e de supervisão, e escassez de relatórios informativos, que acabam por não atender às necessidades das unidades de saúde, embora conheçam a proporção de unidades que trabalhem com TB. Estudos que avaliaram a implantação do PCT em diferentes municípios brasileiros demonstraram que o planejamento no conjunto das ações entre a coordenação do PCT municipal e as unidades de saúde ocorriam de forma esporádica (HEUFEMANN; GONÇALVES; GARNELO, 2013; OLIVEIRA; NATAL, 2007).

Embora os técnicos do PCT avaliado tenham afirmado que a capacitação dos profissionais, na maioria das vezes, é realizada, os resultados referentes ao contexto técnico evidenciaram uma realidade diferente, que se traduz na falta de conhecimentos para ações de vigilância, realização de exames diagnósticos e realização do TDO, entre outras. Estudos que avaliaram o PCT em outros locais também encontraram resultados deficientes em relação à capacitação profissional (HEUFEMANN; GONÇALVES; GARNELO; 2013; OLIVEIRA; NATAL; CAMACHO, 2015). Experiências de outros municípios, que investiram na qualidade e no número de profissionais, através da ampliação da cobertura de ESF, conseguiram proporcionar um aumento nos números de pessoas atendidas e de exames diagnósticos, além de reduzirem os casos novos e abandonos, tendo um relevante papel no controle da TB (MARQUIEVIZ, 2013). Entende-se que, para além da implantação de ESF, é necessário capacitar os profissionais quanto às diretrizes da política da APS, dos programas estratégicos e das ações de saúde elencadas como prioritárias, a exemplo das ações do PCT.

As falhas no gerenciamento de Serviços de Referência e Insumos influenciam sobremaneira o controle e o tratamento da TB. Ações como a distribuição de medicamentos e a análise dos mapas de medicação não acontecem no cotidiano desses serviços. Este fato interfere na organização das unidades de APS, que acabam por não se apropriarem dessas atividades como parte da integralidade do cuidado. A descentralização do 
tratamento pautado no planejamento e na distribuição de medicação para as unidades de APS melhora o desempenho do PCT local (SÁ ET AL., 2012). Este fato pode ser confirmado pelos resultados obtidos em pesquisa sobre a tendência da TB no próprio município, que apontaram para taxas de cura e abandono abaixo do recomendado pelo Ministério da Saúde (SILVA, 2014).

As ações de Vigilância Epidemiológica constituem-se em um conjunto de medidas relevantes para o sucesso do programa. O fato das atividades de vigilância estarem reduzidas ao processo informacional do Sinan pode ser explicado pela relação da obrigatoriedade do envio dessas informações com o repasse de verbas oriundas do Ministério da Saúde. Em outros municípios brasileiros, pesquisas confirmaram que as deficiências de ações de Vigilância Epidemiológica e a inadequação do processo de notificação eram devidas à incapacidade profissional, enquanto problemas com a frequência do fluxo de informações entre as unidades de saúde e a coordenação do PCT, com o envio de notificações, o registro de pacientes e a atualização do livro verde eram oriundos do processo de descentralização do Sinan (FEITOZA ET AL., 2012).

As atribuições dos profissionais de saúde envolvidos com a assistência foram influenciadas de forma significativa pelo contexto organizacional do programa municipal. Embora exista uma estrutura que garante a retaguarda laboratorial, problemas na realização de exames diagnósticos - como o exame radiológico e o tempo entre a coleta de material e a chegada do resultado dos exames - indicam entraves na organização dos serviços e no fluxo das informações. Situações como estas refletem falhas relacionadas à gestão e ao descompromisso por parte dos gestores, comprometendo a continuidade do tratamento e o controle da doença (SÁ ET AL., 2012). A oferta do teste para HIV, quando feita de forma adequada, pode representar mudança em termos de redução de mortalidade por coinfecção HIV/TB, pois a notificação correta dos pacientes contribui para o planejamento de medidas de controle e de assistência ao portador (FEITOZA ET AL., 2012).

As atividades assistenciais do PCT foram prejudicadas pela ausência de um modelo de gestão eficaz, pois doenças como a TB necessitam de gerenciamento do cuidado em longo prazo para, assim, garantirem um prosseguimento da assistência (ASSISETAL., 2012). Descentralizar as ações para a APS e utilizar a ESF como referência do cuidado em rede e como política prioritária de expansão de serviços à população pode ser significativo para o êxito dessa prática. Presume-se que, neste nível de atenção, as ações de vigilância, controle, prevenção e tratamento da TB sejam mais eficazes, pois lidam diretamente com o usuário e seu contexto de vida (BRASIL, 2011; HEUFEMANN; GONÇALVES; GARNELO, 2013).

A realização deficiente do TDO, na rotina de trabalho, pode ser atribuída à centralização de algumas ações assistenciais, à falta de capacitação profissional, à baixa cobertura da ESF e, também, à resistência de alguns profissionais. O TDO é uma estratégia que vai além da simples supervisão da tomada da medicação. Ao conhecer os aspectos sociais e culturais do portador de TB, o profissional pode melhorar o vínculo com este indivíduo, garantindo não somente a promoção da adesão ao tratamento, mas, também, aumento do comprometimento dos profissionais com os usuários. Outro ponto importante do TDO é a possibilidade de redistribuição de poder entre os profissionais de saúde, seja com o enfermeiro coordenando as ações ou com o ACS no contato direto com a comunidade. Entretanto, esta questão tem sido discutida em outros estudos, haja vista o posicionamento de alguns profissionais, favoráveis ao atendimento em serviço especializado sob a alegação de alguns fatores, como a sobrecarga de trabalho por falta de estrutura e planejamento adequados, a escassez e a rotatividade de médicos. Em outros casos, os profissionais consideram que somente 
os usuários em situações de risco social ou psicológico - como, por exemplo, no uso de álcool e drogas e no abandono de tratamento - deveriam ser submetidos ao TDO (CUNHA; CAVALCANTI; COSTA, 2012; CARDOSO ET AL., 2012).

Entre os critérios que mais influenciaram a categoria Prevenção, a visita domiciliar, a busca ativa de novos casos e as atividades de educação em saúde foram pouco utilizadas pelos profissionais de saúde. Esta postura pode ser decorrente da desorganização da gestão ou da falta de conhecimento do profissional em relação aos benefícios desses recursos. Ao serem utilizadas, não apenas para ações de prevenção, mas para fortalecimento de vínculo com a comunidade e identificação das necessidades de saúde, essas práticas facilitam a intervenção nos problemas e demandas identificadas, proporcionando uma clínica de boa qualidade (NOGUEIRA ET AL., 2012).

A opinião dos portadores de TB demonstrou, por um lado, que o PCT tem buscado oferecer condições de acessibilidade para atender às necessidades dessas pessoas. Por outro, no que diz respeito ao diagnóstico e à assistência, percebe-se uma coerência entre a opinião dos portadores e o que foi identificado no restante deste estudo, pois a maioria dos problemas tem sua origem no processo de gestão do programa e no despreparo de alguns profissionais ao executarem ações de tratamento e controle da TB. O fornecimento insuficiente de coletor de escarro e a deficiência na coleta de baciloscopias pode presumir que os profissionais não estejam vigilantes para a TB, suspeitando, inicialmente, de doenças como pneumopatias (LOUREIRO ET AL., 2014). Este fato justifica-se pelo maior número de pedidos de exames radiológicos, que, embora façam parte do diagnóstico da doença, não excluem a baciloscopia, que é fundamental para identificação dos casos de TB pulmonar.

A demora no atendimento, pelo profissional enfermeiro, e a pouca utilização do TDO como estratégia de tratamento e controle da $\mathrm{TB}$, evidenciam que os princípios da APS não estão sendo privilegiados pelo PCT. As condições de acessibilidade não se limitam a características geográficas, mas, também, a condições temporais e culturais, que devem ser consideradas, para que a APS cumpra sua função de prover atenção de qualidade e resolutividade. O TDO, como citado anteriormente, vai além do tratamento medicamentoso, pois, ao aproximar o profissional da realidade do portador de $\mathrm{TB}$, possibilita a construção de vínculo e maior conhecimento de suas necessidades, garantindo que a longitudinalidade e a integralidade sejam contempladas (CUNHA; GIOVANELLA, 2011).

Já os dados da vulnerabilidade social da população do município, medida pela renda e pela escolaridade, demonstraram que esses aspectos podem influenciar de forma direta o contexto da TB. É importante destacar que a vulnerabilidade tem sido reconhecida como um importante componente que envolve o adoecimento por TB (MAFFACCIOLLI ET AL., 2015). Independentemente da escolaridade, a renda baixa priva as pessoas ao acesso a serviços básicos, o que pode facilitar a infecção por TB, configurando a pobreza como um dos principais determinantes sociais da doença (PINHEIRO ET AL., 2013). Mesmo que o município de Divinópolis (MG) apresente indicadores sociais elevados, como o Índice de Desenvolvimento Humano (IDH) e a renda média, identifica-se que, entre os portadores de TB, a baixa renda foi um indicador importante na análise de contexto.

\section{Conclusões}

A avaliação da implantação do PCT de Divinópolis (MG) apontou fragilidades na gestão dos serviços, com prejuízos ao controle e ao tratamento da TB, como a falta de integração entre a coordenação do PCT e as unidades de APS, a ausência de planejamento de ações de supervisão e de políticas de gestão de recursos humanos e centralização na distribuição de medicamentos. Também 
foram verificados problemas nas ações desenvolvidas pelos profissionais da APS, no que diz respeito à fragmentação das práticas, ao preparo para lidar com o portador de $\mathrm{TB}$, bem como para executar atividades de diagnóstico, assistência e prevenção.

As atribuições e ações realizadas na APS, referência do cuidado em rede e política prioritária de expansão de ações e serviços à população, estão abaixo do esperado, sendo influenciadas pela baixa cobertura da ESF e pela descentralização parcial das ações relacionadas ao PCT, contribuindo fortemente para os resultados obtidos neste estudo. Embora os profissionais que participaram da pesquisa terem afirmado que seguiram as recomendações do PNCT e suas normas, percebeu-se que as orientações de vigilância e controle preconizadas não estavam sendo totalmente observadas. É necessário que esses trabalhadores sejam preparados e sensibilizados para assumirem suas responsabilidades frente ao controle da TB. Também é necessário que haja investimentos no aumento e na qualificação de recursos humanos envolvidos.

Com os resultados obtidos, podem-se elencar algumas potencialidades do estudo, como o conhecimento dos pontos críticos que impedem o PCT de alcançar o sucesso no controle da TB, e de propor possíveis soluções aos problemas identificados. Entre as recomendações que demandam maior atenção, podem ser listadas a descentralização, de fato, das ações do PCT, a melhoria da integração entre a coordenação do PCT e as unidades de saúde, a realização das ações de Vigilância Epidemiológica e a capacitação dos recursos humanos, com vistas à melhoria da assistência e da prevenção. A despeito das perdas na amostra, que configuraram uma limitação deste estudo, pode-se destacar que o presente trabalho contribuiu ao apontar, para os gestores, as limitações existentes no processo de organização do PCT municipal, principalmente, em relação à qualificação profissional. Agrega-se, a isso, a abordagem utilizada para analisar o grau de implantação (quantitativa) do programa, que, embora tenha respondido às questões avaliativas, por ser muito normativa, não qualifica as variáveis de contexto que podem explicar os achados.

\section{Colaboradores}

Heuler Souza Andrade - concepção, planejamento, análise e interpretação dos dados e aprovação final do manuscrito. Valéria Conceição Oliveira - revisão crítica do conteúdo e aprovação final do manuscrito. Tarcísio Laerte Gontijo - revisão crítica do conteúdo e aprovação final do manuscrito. Marco Túlio Corrêa Pessôa - revisão da análise estatística e aprovação final do manuscrito. Eliete Albano de Azevedo Guimarães - concepção, planejamento, análise e interpretação dos dados, e aprovação final do manuscrito.

\section{Agradecimentos}

À Universidade Federal de São João Del Rei; à Secretaria Municipal de Saúde de Divinópolis (MG), Brasil; e à Secretaria de Estado de Saúde de Minas Gerais, Brasil. 


\section{Referências}

ASSIS, E. G. et al. A coordenação da assistência no controle da tuberculose. Rev. Esc. Enferm. USP, São Paulo, v. 46, n. 1, p. 111-18, fev. 2012.

BRASIL. Ministério da Saúde. Secretaria de Vigilância em Saúde. Boletim Epidemiológico. v. 46, n. 9, 2015.

Disponível em: <http://u.saude.gov.br/images/ pdf/2015/marco/25/Boletim-tuberculose-2015.pdf>. Acesso em: 21 out. 2016.

Ministério da Saúde. Secretaria de Vigilância em Saúde. Departamento de Vigilância Epidemiológica. Manual de Recomendações para o Controle da Tuberculose no Brasil. Brasília, DF: Ministério da Saúde, 2011. (Série A. Normas e Manuais Técnicos).

CHAMPAGNE, F. et al. A avaliação no campo da saúde: conceitos e métodos. In: BROUSSELLE, A. et al (Org.). Avaliação em saúde: conceitos e métodos. Rio de Janeiro: Fiocruz, 2011, p. 41- 60.

CARDOSO, G. C. P. et al. A conformidade das ações do tratamento diretamente observado para tuberculose na perspectiva dos profissionais de duas unidades de saúde da cidade do Rio de Janeiro. Cad. Saúde Colet., Rio de Janeiro, v. 20, n. 2, p. 203-10, 2012.

CUNHA, E. M.; GIOVANELLA, L. Longitudinalidade/ continuidade do cuidado: identificando dimensões e variáveis para a avaliação da Atenção Primária no contexto do sistema público de saúde brasileiro. Ciênc. Saúde Colet., Rio de Janeiro, v. 16, supl. 1, p. 1029-42, 2011.

CUNHA, N. V.; CAVALCANTI, M. L. T.; COSTA, A. J. L. Diagnóstico situacional da descentralização do controle da tuberculose para a Estratégia Saúde da Família em Jardim Catarina - São Gonçalo (RJ), 2010. Cad. Saúde Colet., Rio de Janeiro, v. 20, n. 2, p. 177-87, abr. 2012.

DARA, M.; COLOMBANI, P.; PETROVA, B. R. Minimum package for cross-border TB control and care in the WHO European region: a Wolfheze consensus statement. Eur. Respir. J., Sheffield, v. 40, n.
5, p. 1081-1090, nov. 2012.

FEITOZA, D. S. et al. Vigilância Epidemiológica no contexto do Programa de Controle da Tuberculose: limites e possibilidades. Rev. Rene, Fortaleza, v. 13. n. 5, p. 1066-74, 2012.

GUTIERREZ, S. E. Avaliação da implantação do Programa de Controle da Tuberculose em unidades de saúde da atenção básica nas regionais norte e sul de Cuiabá/MT, 2010. 133 f. 2012. Dissertação (Mestrado em Saúde Pública) - Fundação Oswaldo Cruz, Escola Nacional de Saúde Pública Sergio Arouca, Rio de Janeiro, 2012.

\section{HEUFEMANN, N. E. C.; GONÇALVES, M. J. F.;}

GARNELO, M. L. Avaliação do programa de controle da tuberculose em cenário Amazônico: desafios em Coari. Acta Amaz., Petrópolis, MA, v. 43, n. 1, p. 33-42, 2013.

LOUREIRO, R. B. et al. Acesso ao diagnóstico da tuberculose em serviços de saúde do município de Vitória, ES, Brasil. Ciênc. Saúde Colet., Rio de Janeiro, v. 19, n. 4, p. 1233-44, abr. 2014.

MAFFACCIOLLI, R. et al. A utilização da noção de vulnerabilidade na produção de conhecimento sobre tuberculose: revisão integrativa. Rev. Gaúcha Enferm., Porto Alegre, v. 36, n. esp., p. 247-253, 2015.

MARQUIEVIZ, J. et al. A Estratégia de Saúde da Família no controle da tuberculose em Curitiba (PR). Ciênc. Saúde Colet., Rio de Janeiro, v. 18, n. 1, p. 265-271, jan. 2013.

NOGUEIRA, J. A. et al. Vínculo e acesso na estratégia saúde da família: percepção de usuários com tuberculose. Rev. Rene., Fortaleza, v. 13, n. 4, p. 784-93, 2012.

OLIVEIRA, L. G. D.; NATAL, S.; CAMACHO, L. A. B. Análise da implantação do Programa de Controle da Tuberculose em unidades prisionais no Brasil. Cad. Saúde Púb., Rio de Janeiro, v. 31, n. 3, p. 543-554, mar. 2015. 
OLIVEIRA, L. G. D.; NATAL, S. Avaliação de implantação do Programa de Controle da Tuberculose no município de Niterói/RJ. Rev. Bras. Pneumol. Sanit., Rio de Janeiro, v. 15, n. 1, p. 29-38, 2007.

PINHEIRO, R. S. et al. Determinantes sociais e autorrelato de tuberculose nas regiões metropolitanas conforme a Pesquisa Nacional por Amostra de Domicílios, Brasil. Rev. Panam. Salud. Publ., Washignton, v. 34, n. 6, p. 446-51, dez. 2013.

PONCE, M. A. Z. et al. Diagnóstico da tuberculose: desempenho do primeiro serviço de saúde procurado em São José do Rio Preto, São Paulo, Brasil. Cad. Saúde Púb., Rio de Janeiro, v. 29, n. 5, p. 945-954, maio 2013.

SÁ, L. D. et al. Cuidado ao doente de tuberculose na Estratégia Saúde da Família: percepções de enfermeiras. Rev. Esc. Enferm. USP, São Paulo, v. 46, n. 2, p. 356-63, abr. 2012.

SCATENA, L. M. et al. Dificuldades de acesso a serviços de saúde para diagnóstico de tuberculose em municípios do Brasil. Rev. Saúde Púb., São Paulo, v. 43, n. 3, p. 389-97, jun. 2009.

SILVA, D. M. et al. Avaliação de desempenho de Serviços da Atenção Básica para o tratamento da tuberculose. Rev. Esc. Enferm. USP, São Paulo, v. 48, n. 6, p. 1044-53, dez. 2014.

SILVA, M. A. Tendência da Tuberculose no município de Divinópolis - MG, de 2002 A 2012. 96 f. 2014.

Dissertação (Mestrado) - Universidade Federal de São João Del Rei (UFSJ), Divinópolis, 2014.

VILLA, T. C. S. et al. Diagnóstico oportuno da tuberculose nos serviços de saúde de diversas regiões do Brasil. Rev. Latino-Am. Enfermagem, Ribeirão Preto, v. 21 , n. esp., p. 190-98, fev. 2013.

\section{WORLD HEALTH ORGANIZATION (WHO).}

Global tuberculosis report 2014. Geneva: WHO,

2014. Disponível em: <http://apps.who.int/iris/

bitstream/10665/137094/1/9789241564809_eng.pdf>.

Acesso em: 21 out. 2016.

YIN, R. K. Estudo de Caso: planejamento e métodos. 4. ed. São Paulo: Bookman, 2010

\footnotetext{
Recebido para publicação em janeiro de 2016

Versão final em agosto de 2016

Conflito de interesses: inexistente

Suporte financeiro: Pesquisa financiada pela Fundação Universidade Federal de São João Del Rei / Pró-Reitoria de Pesquisa e Pós-Graduação (Prope)
} 